differential extinction rates over blocks of trials as a function of complexity and set.

The analysis of variance (specific GSRs) reveals that complexity $[F(2,24)=16.83$, $\mathrm{p}<.001]$ and set $[\mathrm{F}(1,24)=8.91, \mathrm{p}<.01]$ effects were significant, although there was no interaction between the two main effects. The response curves, as per Fig. 1, clearly demonstrate that the slopes of the extinction curves in the three complexity conditions differ markedly. It is evident that extinction rates are more rapid under less complex problems and that, when unsolvable set precedes extinction phase, the extinction rates are slower when compared to those in solvable set conditions.

\section{DISCUSSION}

Although in a previous study (Pishkin, Shurley, \& Wolfgang, 1967) set and complexity did not influence extinction of the specific GSRs, present findings clearly reveal significant complexity and set effects. In the unsolvable set condition of the earlier study (Pishkin, Shurley, \& Wolfgang, 1967) complexity was the same as in the Phase 2 , i.e., one, three, or five irrelevant dimensions; in the present study complexity of the set phase was always five irrelevant dimensions. Thus, high complexity (five dimensions) and unsolvable set interacted sufficiently to produce marked differentiation in extinction phases between the experimental conditions. It is also possible that the high complexity conditions (five irrelevant dimensions) during extinction were more similar to the original conditioning phase, which also had five irrelevant dimensions, resulting in slower extinction through a tendency for generalization from condition. ing to extinction.

In brief, the findings demonstrate increasing inhibition of extinction of conditioned GSR as a function of increasing complexity of concept identification problems, as well as a function of the antecedent set conditions. These data further support the view, derived from cybernetic theory of brain function, that all of the brain's "computing" capacity is used for current purposes. The essential psychobiological task of clearing out, here, in the waking state, no-longer-needed functional programs in the brain, Spence's (1966) theoretical "inhibitory set" or the extinction of a GSR appears to be delayed or inhibited when the structure of the set and of the cognitive task "masks" or does not permit the braincomputer to strike a quantitative probability balance in detection of changes in response-stimulus contingencies. Furthermore, it is noteworthy that the results show a reliable relationship between information load, i.e., number of irrelevant dimensions in concept identification, and extinction rates of GSR. It is clear that Spence's (1966) theorizing related to influence of cognitive

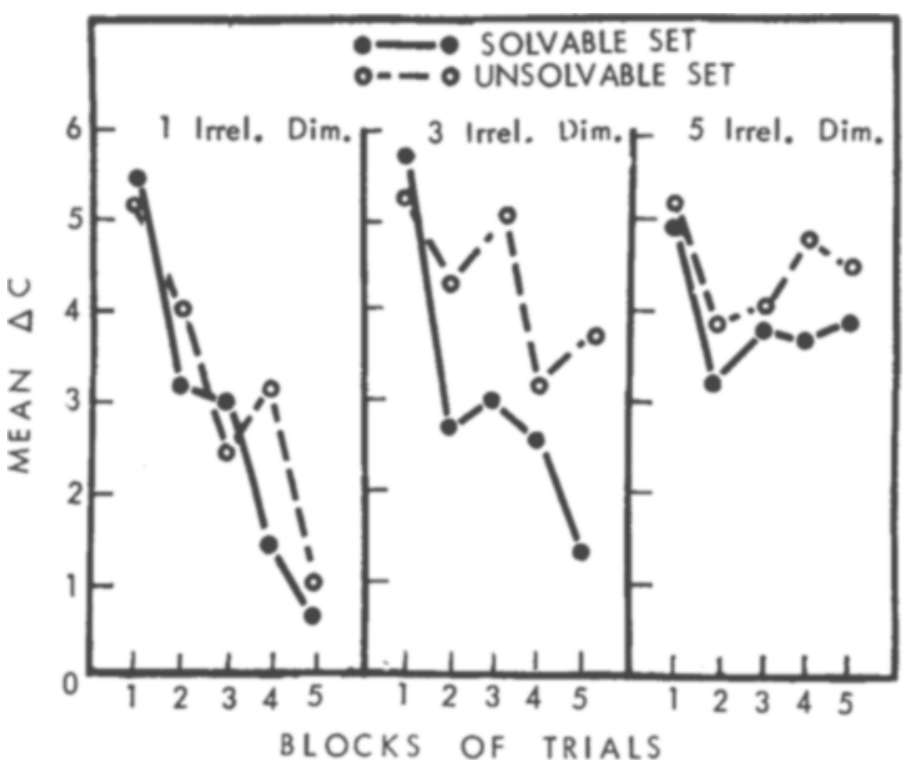

Fig. 1. Mean conductance changes in micromhos as functions of one, three, or five bits of irrelevant information and solvable or unsolvable sets. The data are represented in five trial blocks, each consisting of six ex tinction trials, over a 30-min period.

factors and sets on extinction data of conditioned eye-blink is supported strongly by the present findings with extinction of conditioned GSR responses.

\section{REFERENCES}

EDELBERG, R. Electrical properties of the skin. In C. C. Brown (Ed.), Methods in psychophysiology. Baltimore: The Williams \& Wilkins Co., 1967. Pp. 1-53.

ESTES, W. K., \& STRAUGHAN, J. W. Analysis of a verbal conditioning situation in terms of statistical learning theory. Joumal of Experimental Psychology, 1954, 47, 225-234.

PISHKIN, V. Dimension availability with antecedent success or failure in concept identification. Psychonomic Science, 1965, 2, 69-70.
PISHKIN, V., \& SHURLEY, J. T. Electrodermal and electromyographic parameters in concept identification. Psychophysiology, 1968, 5, $112-118$.

PISHKIN, V., SHURLEY, J. T., \& WOLFGANG, A. Stress. Archives of General Psy chiatry, 1967, $16,471-477$

SPENCE, K. W. Cognitive and drive factors in the extinction of the conditioned eye blink in human subjects. Psychological Review, 1966, 73, 445-458.

SPENCE, K. W., HOMZIE, M. J., \& RUTLEDGE, E. F. Extinction of the human eyelid $C R$ as a function of the discriminability of the change from acquisition to extinction. Journal of Experimental Psychology, 1964, 67, 545-552. NOTE

1. The authors are indebted to Robert Edelberg for his valuable suggestions.

\title{
Decrease in polarity of semantic differential ratings as a function of previous trials with similar materials
}

WILLIAM E. GUMENIK, The University of
Toledo, Toledo, Ohio 43606

Eighty eight Ss rated 10 good words and 88 Ss, 10 bad words on a 7-point good-bad semantic differential scale. In both cases, the polarity of the ratings decreased as a direct linear function of the number of words previously rated.
Osgood (1957, pp. 84-85) assumed that semantic differential ratings of an item are independent of the meaning of other items on the test form and thereby unaffected by anchor and central tendency effects. He reported an unpublished study by Aiken which supported this assumption. Investigators, on the basis of this assumption of independence, have often, as a matter of convenience, presented the items to be judged in a set order, even in the case of 


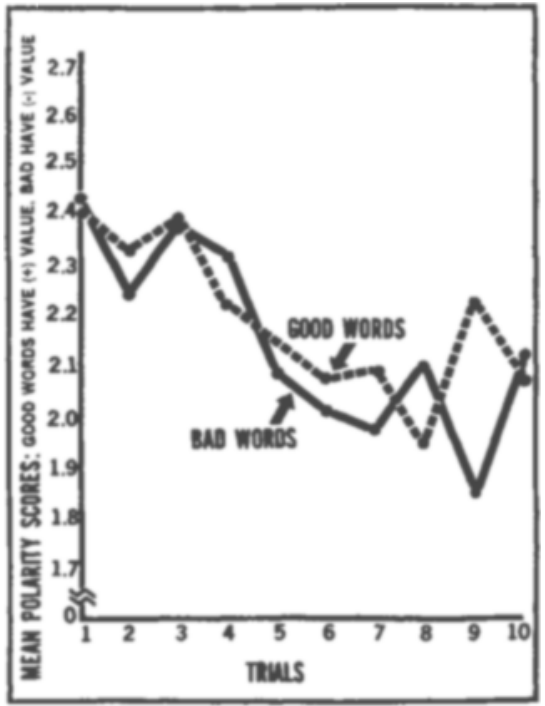

Fig. 1. Mean polarity scores for good and bad words on the 10 trials.

determining norms (i.e., Wilcox \& Pollio, 1966).

AL theory (Helson, 1964) has been used to account for several judgmental and perceptual phenomena, including that of semantic satiation, i.e., reduction in polarity of semantic differential ratings of repeated stimuli (Amster, 1964). According to the theory, judgments are made in reference to AL which is a weighted log mean of all focal, background, and past stimuli. The degree of polarization of stimulus is a direct function of its distance from AL. Presentation of highly polarized stimuli should result in a shift in AL roward these stimuli with a resulting reduction in polarity of subsequently presented similar stimuli. It would therefore be expected that as number of similar items rated on semantic differential scales increase, the polarity of the ratings should decrease.

\section{METHOD}

The Ss were 176 undergraduates randomly divided into two treatment groups of $88 \mathrm{Ss}$ each. The Ss were tested in groups ranging in size from 14 to $48 \mathrm{Ss}$, both treatment groups being equally represented on each testing occasion.

Standard written instructions similar to those suggested by Osgood (1957, pp. 82-84) with some slight modifications were given to $S$ to read and were read aloud by $E$. The fifth sentence before the end of the instructions, "Make each item a separate and independent judgment," was stressed by $E$ in the reading of the instructions. $S$ then received a booklet with either 10 good or 10 bad words in it, depending on treatment group. Each word appeared on a separate page with a seven-point good-bad scale under it. The words were selected from previously established norms, based on 93
University of Toledo undergraduates, for 140 common monosyllabic words. The mean polarity scores, on the norms, for the words used in this study were $+2.41, \sigma=.14$ and $-2.23, \sigma=.15$ for the 10 good and 10 bad words, respectively (on a seven-point scale ranging from +3 for good to -3 for bad). The words were presented in a different random order for each $S$.

\section{RESULTS}

Mean polarity scores for good and bad words on the 10 trials are presented in Fig. 1. The mean polarity scores on the ordinate have a (+) value for the good words and a (-) value for the bad words. Superimposition of positive and negative polarity scores in this manner makes it possible to observe how the good words and bad words parallel each other in reduction of polarity over trials. If such superimposition had not been done, the curves, of course, would be widely separated and would converge over trials. ANOV revealed a significant Meaning by Trials interaction $[F(9,1566)=4.75, p<.001]$, as well as a meaning main effect $[F(1,174)=2542.83$, $\mathrm{p}<.001]$. Simple effects for good words revealed a significant trials effect $[F(9,1566)=2.13, p<.05]$, and tests for trend showed the linear component to be significant $[F(1,1566)=11.76, p<.001]$, while the nonlinear components were not. Similarly, simple effects for bad words revealed a significant trials effect $[F(9,1566)=3.67, p<.001]$, and again tests for trend showed the linear component to be significant $[F(1,1566)=20.15$, $\mathrm{p}<.001]$, while the nonlinear components were not. Despite the reduction in polarity of ratings over trials, placement of a good word in any of the three bad categories $(-1$ to -3 ) or of a bad word in any of the three good categories $(+1$ to +3$)$ was very rare. It never exceeded $2 \%$ of the Ss on any one trial for either group. On the other hand, use of the neutral $(0)$ category increased, for the good words, from $5 \%$ of the Ss on Trial 1 to $12 \%$ on Trial 10 , and, for the bad words, from $1 \%$ on Trial 1 to $10 \%$ on Trial 10 .

$$
\text { DISCUSSION }
$$

There was a significant reduction in polarity of semantic differential ratings as a direct linear function of the number of previously rated similar words. The results are in agreement with $\mathrm{AL}$ theory (Helson, 1964). As AL shifts toward the highly polarized words, one would expect a decrease in the polarity of the ratings and an increase in the use of the neutral category, which occurred. Since AL would approach but not exceed the polar positions of the presented words, no increase would be anticipated in Ss placing good words in bad categories or bad words in good categories. No more than a minimal number of Ss did this on any trial.
Other explanations of the present results are of course possible. For example, one might postulate that Ss have a tendency not to continually rate different items in the same category. Since the words were highly polarized, the categories which were least used initially were the less polarized categories, the neutral category, and those on the other side of the bipolar scale. Use of these categories on later trials would, of course, reduce polarity of the ratings. However, categories on the other side of the bipolar scale were rarely used on any trial. While it would seem reasonable that, despite this tendency, Ss might be reluctant to rate a highly polarized good word as bad or a highly polarized bad word as good, it is not clear why Ss than demonstrated a willingness to categorize highly polarized good or bad words as neutral on later trials. Also, according to this explanation, one might expect Ss who rated the initial word less extremely $(+2$ or -2 instead of +3 or -3$)$ would show greater polarity of later ratings than those Ss who used the most polarized categories initially. Instead, it was found that Ss who initially used less polarized (2) ratings showed lower polarity of ratings on later trials than Ss who initially used the most polarized (3) ratings. This tendency was significant for good words $[t(77)=2.76, p<.01]$ but not for bad words.

Osgood's (1957, pp. 84-85) assumption that semantic differential ratings of an item are independent of the other items on the test form, and thereby unaffected by anchor and central tendency effects, was not supported. It is true that sequential presentation of several stimuli having similar semantic differential scores is extreme and would rarely occur in usual practice. Also, only one semantic differential scale was used, whereas usually several scales are used for each item. However, it is apparent from the present results that there are substantial effects of previously rated items on later semantic differential ratings. Care must be taken, in using semantic differential ratings, to eliminate any bias which might be attributable to this factor.

\section{REFERENCES}

AMSTER, H. Semantic satiation and generation: Learning? Adaptation? Psychological Bulletin, 1964, 62, 273-287.

HELSON, H. Adaptation-level theory. New York: Harper and Row, 1964.

OSGOOD, C. E., SUCI, G. S., \& TANNENBAUM, $\mathrm{P} . \mathrm{H}$. The measurement of meaning. Urbana: University of Illinois Press, 1957.

WILCOX, R. C., \& POLLIO, H. R. Semantic ratings for 630 words and some correlated associative properties. University of Tennessee Technical Report No. 3,1966.

$$
\text { NOTE }
$$

1. The author wishes to thank Michael Brumet for his assistance in assembling the materials and tabulating the data. 